\title{
An Appraisal of the 2018 Guidelines for the Early Management of Patients with Acute Ischemic Stroke
}

\author{
Ashutosh P. Jadhav ${ }^{a}$ Maxim Mokin ${ }^{b}$ Santiago Ortega-Gutierrez ${ }^{c}$ \\ Diogo Haussen $^{d}$ David Liebeskind ${ }^{\mathrm{e}}$ Raul Nogueira $^{d}$ Tudor Jovin $^{a}$ \\ Italo Linfante ${ }^{f}$ on behalf of the Society of Vascular and Interventional \\ Neurology (SVIN)

\begin{abstract}
aDepartment of Neurology and Neurosurgery, University of Pittsburgh Medical Center, Pittsburgh, PA, USA; ${ }^{b}$ Department of Neurology and Neurosurgery, University of South Florida, Tampa, FL, USA; ${ }^{c}$ Department of Neurology, Radiology and Neurosurgery, University of lowa, lowa City, IA, USA; d Department of Neurology and Neurosurgery, Emory University, Atlanta, GA, USA; ${ }^{\text {DD }}$ epartment of Neurology, University of California Los Angeles, Los Angeles, CA, USA; ${ }^{\mathrm{f}}$ Miami Cardiac and Vascular Institute and Baptist Neuroscience Center, Miami, FL, USA
\end{abstract}

On January 24, 2018 the American Heart Association (AHA) released the Guidelines for the Early Management of Patients with Acute Ischemic Stroke [1]. The intended purpose set forth by the writing committee was to provide recommendations to clinicians caring for adult patients with acute ischemic stroke. The target audience included prehospital care providers, allied health professionals, and hospital administrators. Acute care of suspected large vessel occlusion (LVO) patients and its influence on prehospital triage and stroke systems of care represent key topics of this guideline. The aim of this commentary is to highlight areas where, in the opinion of the authors, the guidelines are inconsistent and fail to adequately address the relevance of the available data to real-world clinical practice.

\section{Prehospital Triage}

The most important reason that acute ischemic stroke patients may not be treated by revascularization therapies is time delay in the prehospital phase. The recent strong evidence supporting mechanical thrombectomy to treat patients with LVO has created an important dilemma in the organization of systems of care as in the ideal world, both intravenous (IV) alteplase and thrombectomy need to occur without any time delays. The latest AHA guidelines favor IV thrombolysis over thrombectomy despite the evidence that in patients with 
LVO who present with most devastating strokes, thrombectomy is the only treatment with a high chance of neurologic recovery.

\section{Emphasis on Delivering IV Alteplase}

Current guidelines recommend patient transportation to the nearest hospital with IV alteplase capability, a recommendation that has been carried over from the AHA guidelines since 2013 before mechanical thrombectomy was considered standard of care for LVO (COR 1 , LOE B-NR). It is also stated that when several IV alteplase-capable hospital options exist within a geographic area, the benefit of bypassing the closest facility to bring the patient to the one that offers the higher level of stroke care, including mechanical thrombectomy, is uncertain (COR IIb, LOE B-NR). Furthermore, due to the lack of randomized trials and limited predicted performance of stroke severity scales in the prehospital setting, guidelines prefer to emphasize the role of IV alteplase and consider direct transport to a comprehensive stroke center if the transport delay is $<15$ additional minutes compared with the travel time to the closest stroke-ready hospital. This conclusion is derived from a non-randomized analysis from the GWTG national registry that suggests that for every 15 min delay of IV alteplase, there is a decrease likelihood of achieving independence in ambulation (OR: 1.04; 95\% CI 1.03-1.05) and discharge home (OR, 1.03; 95\% CI 1.02-1.04) in all stroke subtypes [2].

We agree with the authors that there is an immediate need for further research in this topic. Several ongoing trials are addressing the issues of optimal prehospital patient triage (RACE-CAT) and the efficacy of combined reperfusion therapy versus mechanical thrombectomy alone (SWIFT DIRECT, MR-CLEAN 2). We also understand that the implementation of this algorithm represents minimal disruption on the current systems of care, but we believe that the recommendations might have involuntarily neglected data that might argue against this approach. Intravenous IV alteplase treatment is effective in acute stroke patients, especially with mild and moderate symptoms. The efficacy of IV tPA in terms of arterial recanalization in the first hours progressively drops as the burden of the occlusive clot increases. While the recanalization rate of an M2 occlusion is around $45 \%$, the recanalization rates of M1 or terminal ICA occlusions drop to 30 and 10\%, respectively. Furthermore, in the HERMES collaboration meta-analysis, outcomes were compared in patients who underwent thrombectomy without IV alteplase to those who received combined therapy. The good outcome in the 1,080 patients eligible for IV tPA was $46.4 \%$ with thrombectomy and $27 \%$ in the control group (cOR $=2.45$; 95\% CI 1.68-3.57), while the good outcomes in the 188 IV tPA-ineligible patients were similar (thrombectomy $43.5 \%$ vs. $22.3 \%$ control; cOR $=2.43$; $95 \%$ CI 1.30 $4.55)$ without heterogeneity in the intervention treatment effect $(p=0.43)$ for interaction. Additionally, in patients that present beyond the therapeutic IV tPA time window, the guidelines do not adequately acknowledge the collective evidence supporting thrombectomy from DAWN and DEFUSE 3. In this late time window population, patients should be re-routed towards thrombectomy-capable centers as this paradigm gives this population the best chance of rapidly being treated with a very effective intervention.

\section{Worse Outcomes in Transfer Patients}

A subgroup analysis of the SWIFT PRIME trial demonstrated that patients treated under the current drip and ship paradigm had a significant delay from symptom onset to groin puncture when compared to patients directly transferred to endovascular-capable centers (275 vs. $179 \mathrm{~min}$ ) [3]. The major cause of delay among patients who received IV alteplase before transfer was the prolonged arrival time from referral hospitals to the emergency department. More importantly, for every 60 min delay in the treatment group after $35.5 \mathrm{~min}$ of treatment with endovascular therapy (EVT), there was a $20 \%$ lower likelihood of functional independence. This analysis fails to account for the many patients that were no longer 
candidates for EVT by the time they arrived at the endovascular-capable center due to interval stroke progression. Similar results were obtained from analysis of the MR CLEAN trial and the STRATIS registry $[4,5]$. Indeed, a small non-randomized observational study demonstrated that one out of three patients with LVO with initial favorable imaging profile became ineligible for endovascular thrombectomy during inter-hospital transfer based on ASPECTS criteria [6]. In this study, except for NIHSS severity, no other baseline factors could identify which patients were at risk for ASPECTS deterioration during inter-hospital transfer. Although this study is small, the data are remarkably important given the fact that those thrombectomynon-eligible patients are rarely consistently collected in a randomized trial. Finally, a highquality prospective registry (STRATIS) of 984 patients demonstrated that inter-hospital transfer was associated with a significant delay in onset-to-recanalization time (202.0 vs. $311.5 \mathrm{~min} ; p<0.001$ ) and decreased likelihood of good functional outcome (60 vs. 52.2\%; OR 1.38; 95\% CI 1.06-1.79) in patients with LVO [5]. Interestingly, the subgroup of patients who were transferred and received IV alteplase not only had a longer median onset-to-revascularization time of $192 \mathrm{~min}$, but also significant delays in door-to-IV alteplase times (54.5 vs. 37 $\mathrm{min}$ ) and median tPA-to-transportation delays between IV alteplase and departure from the initial hospital (median, $47 \mathrm{~min}$; IQR, 27-85), and length of transport time (median, $35 \mathrm{~min}$; IQR, 21-58). In the same study, a hypothetical bypass modelling revealed that if bypass is limited to a 20 -mile radius, the delay for IV alteplase would be 7 min and thrombectomy would have started 94 min earlier.

While no randomized trial data exist to support a firm recommendation on the acceptable delay in arrival at a stroke center when considering re-routing a comprehensive stroke center, there is considerable data demonstrating that triaging patients to the non-endovascularcapable hospitals might translate in substantial delays on delivering the most effective available treatment against devastating strokes.

\section{Endovascular Therapy}

\section{Late Time Window Patients}

Demonstrating efficacy of EVT up to $24 \mathrm{~h}$ from stroke symptom onset is undoubtedly one of the biggest breakthroughs in the field of acute stroke management in 2017 (section 2.2-12). A new recommendation (COR 1 and LOE A) from the guidelines indicate that patients with arterial ischemic stroke within 6 to $16 \mathrm{~h}$ of last known normal who have LVO in the anterior circulation and meet DAWN or DEFUSE eligibility should receive mechanical thrombectomy (section 3.7.-7). However, for selected patients within 6 to $24 \mathrm{~h}$ of the time last known normal who have LVO in the anterior circulation and meet DAWN criteria, mechanical thrombectomy is reasonable (COR IIa; LOE B-R). These two recommendations create an impression of inconsistency. Specifically, the DAWN trial did not include patients with an NIHSS of 6-9 and yet this group is included in the class I recommendation based solely on the DEFUSE 3 trial [7]. Notably, the benefit in DEFUSE 3 for the pre-specified NIHSS 6-9 category did not reach statistical significance for functional outcome (95\% CI 1.43 [0.65-3.15]), whereas the benefit in DAWN for the 12-24-hour time window did [7, 8]. Based on this rationale, we believe it is reasonable to consider that both subgroups, NIHSS 6-9 and NIHSS 16-24 h from last known normal, should be class I recommendation. It would be helpful to understand the scientific justification for class I recommendation for the NIHSS 6-9 subgroup and the class Ila recommendation for the 16-24-hour subgroup.

The guidelines limit indications for thrombectomy within 6-24 h only to patients who meet inclusion criteria for DEFUSE 3 and DAWN. However, the REVASCAT trial showed the benefit of thrombectomy up to $8 \mathrm{~h}$ and the ESCAPE trials up to $12 \mathrm{~h}$, with neither trial 
mandating the use of perfusion-based criteria $[9,10]$. In addition, rates of clinical outcomes from real-world registries such as STRATIS (0-8 h), NASA (no time limit), and TREVO (no time limit) replicated the rates of outcome from the randomized trials [11-13]. Failure to acknowledge the benefit of thrombectomy for a broader population of patients with LVO presenting with stroke symptoms beyond $6 \mathrm{~h}$ will undoubtedly limit their chances of receiving this highly effective and currently the only available treatment. A recently published analysis from a DAWN trial-participating single comprehensive stroke center helps estimate how many patients presenting with symptoms beyond the 6-hour window will fall outside of the DAWN and DEFUSE 3 selection criteria [14]. Of 792 patients arriving within the 6-24-hour time window, 174 had NIHSS $\geq 6$ and anterior circulation LVO. Of those 174 patients, $58 \%$ (101) patients were not DAWN or DEFUSE eligible. Therefore, under the current AHA guidelines, more than half of the patients with LVO and symptom onset beyond $6 \mathrm{~h}$ would be ineligible for thrombectomy, thus depriving them of any chance for meaningful recovery.

\section{Posterior Circulation Patients}

Patients with posterior circulation stroke after $6 \mathrm{~h}$ of stroke onset would be excluded from EVT based on the latest AHA guidelines. While there are currently no randomized controlled trial data to support EVT outside of strict DAWN and DEFUSE 3 criteria, there is certainly no evidence to argue against such therapy. Bearing in mind all available up-to-date evidence from registries and non-randomized studies, EVT may be reasonable for carefully selected patients within 6-24 h of symptom onset who do not meet the criteria from the DAWN and DEFUSE 3 trials, including posterior circulation strokes with an LVO. It is time to acknowledge that we have reached a point in the treatment of acute ischemic stroke where the amount of high-level data from the recent trials of EVT has led to a loss of equipoise between medical and endovascular stroke therapy. Patients and their families will no longer agree to be randomized just for the sake of science if they are provided with accurate data on the safety, efficacy, and availability of EVT. In addition, given the current data, we, as a community of stroke and endovascular providers, should attempt to do the best for the patients and families and not just to attempt to pursue level 1 evidence to treat this dismal disease that has currently a therapy that drastically could help. This is well exemplified in the slow enrollment of the BASICS trial, which since 2011 has only randomized 200 patients (as of April 19, 2018) [15].

\section{Populations Not Studied in Randomized Controlled Trials}

All randomized trials of modern approaches to EVT published since 2014 have shown the thrombectomy procedure to be safe and highly effective for the treatment of stroke due to emergent LVO. Real-world evidence indicates that patients who do not meet top tier evidence criteria (including tandem lesions) for EVT still demonstrate high rates of good clinical outcome and low complication rates with thrombectomy $[12,16,17]$. Depending on the sets of selection criteria, between $3 \%$ (the most restrictive criteria) and 20\% (the least restrictive criteria) of patients with LVO may be potentially eligible for EVT $[18,19]$. Withholding EVT from patients who do not meet all selection criteria from the recent randomized trials will negatively affect outcomes of thousands of patients with acute ischemic stroke.

\section{Conclusion}

The authors feel that the new AHA guidelines did not fully recognize the overwhelming benefit for thrombectomy in a broad population of patients with LVO with important implications for prehospital and hospital policies, clinical decision making, and ultimately patient care. In particular, given the poor natural history of LVO disease, with increasing data 
supporting the safety and efficacy of EVT along with the harm of treatment delays, it is our opinion that the new guidelines should more strongly align their recommendations with the importance of timely triage and treatment of this vulnerable population with the worst clinical outcomes when reperfusion is delayed or not performed.

\section{References}

1 Powers WJ, Rabinstein AA, Ackerson T, Adeoye OM, Bambakidis NC, Becker K, et al.; American Heart Association Stroke Council. 2018 Guidelines for the Early Management of Patients With Acute Ischemic Stroke: A Guideline for Healthcare Professionals From the American Heart Association/American Stroke Association. Stroke. 2018 Mar;49(3):e46-110.

2 Saver JL, Fonarow GC, Smith EE, Reeves MJ, Grau-Sepulveda MV, Pan W, et al. Time to treatment with intravenous tissue plasminogen activator and outcome from acute ischemic stroke. JAMA. 2013 Jun; 309(23): 2480-8.

3 Goyal M, Jadhav AP, Bonafe A, Diener H, Mendes Pereira V, Levy E, et al.; SWIFT PRIME investigators. Analysis of Workflow and Time to Treatment and the Effects on Outcome in Endovascular Treatment of Acute Ischemic Stroke: Results from the SWIFT PRIME Randomized Controlled Trial. Radiology. 2016 Jun;279(3):888-97.

4 Venema E, Boodt N, Berkhemer OA, Rood PP, van Zwam WH, van Oostenbrugge RJ, et al.; MR CLEAN investigators. Workflow and factors associated with delay in the delivery of intra-arterial treatment for acute ischemic stroke in the MR CLEAN trial. J Neurointerv Surg. 2018 May;10(5):424-8.

5 Froehler MT, Saver JL, Zaidat 00, Jahan R, Aziz-Sultan MA, Klucznik RP, et al.; STRATIS Investigators. Interhospital Transfer Before Thrombectomy Is Associated With Delayed Treatment and Worse Outcome in the STRATIS Registry (Systematic Evaluation of Patients Treated With Neurothrombectomy Devices for Acute Ischemic Stroke). Circulation. 2017 Dec;136(24):2311-21.

6 Mokin M, Gupta R, Guerrero WR, Rose DZ, Burgin WS, Sivakanthan S. ASPECTS decay during inter-facility transfer in patients with large vessel occlusion strokes. J Neurointerv Surg. 2017 May;9(5):442-4.

7 Nogueira RG, Jadhav AP, Haussen DC, Bonafe A, Budzik RF, Bhuva P, et al.; DAWN Trial Investigators. Thrombectomy 6 to 24 Hours after Stroke with a Mismatch between Deficit and Infarct. N Engl J Med. 2018 Jan; 378(1):11-21.

8 Albers GW, Marks MP, Kemp S, Christensen S, Tsai JP, Ortega-Gutierrez S, et al.; DEFUSE 3 Investigators. Thrombectomy for Stroke at 6 to 16 Hours with Selection by Perfusion Imaging. N Engl J Med. 2018 Feb; 378(8):708-18.

9 Jovin TG, Chamorro A, Cobo E, de Miquel MA, Molina CA, Rovira A, et al.; REVASCAT Trial Investigators. Thrombectomy within 8 hours after symptom onset in ischemic stroke. N Engl J Med. 2015 Jun;372(24):2296-306.

10 Goyal M, Demchuk AM, Menon BK, Eesa M, Rempel JL, Thornton J, et al.; ESCAPE Trial Investigators. Randomized assessment of rapid endovascular treatment of ischemic stroke. N Engl J Med. 2015 Mar;372(11):1019-30.

11 Mueller-Kronast NH, Zaidat O0, Froehler MT, Jahan R, Aziz-Sultan MA, Klucznik RP, et al.; STRATIS Investigators. Systematic Evaluation of Patients Treated With Neurothrombectomy Devices for Acute Ischemic Stroke: Primary Results of the STRATIS Registry. Stroke. 2017 Oct;48(10):2760-8.

12 Zaidat 00, Castonguay AC, Nogueira RG, Haussen DC, English JD, Satti SR, et al. TREVO stent-retriever mechanical thrombectomy for acute ischemic stroke secondary to large vessel occlusion registry. J Neurointerv Surg. 2017.

13 Zaidat 00, Castonguay AC, Gupta R, Sun CH, Martin C, Holloway WE, et al. North American Solitaire Stent Retriever Acute Stroke registry: post-marketing revascularization and clinical outcome results. J Neurointerv Surg. 2014 Oct;6(8):584-8.

14 Jadhav AP, Desai SM, Kenmuir CL, Rocha M, Starr MT, Molyneaux BJ, et al. Eligibility for Endovascular Trial Enrollment in the 6- to 24-Hour Time Window: Analysis of a Single Comprehensive Stroke Center. Stroke. 2018 Apr;49(4):1015-7.

15 van der Hoeven EJ, Schonewille WJ, Vos JA, Algra A, Audebert HJ, Berge E, et al.; BASICS Study Group. The Basilar Artery International Cooperation Study (BASICS): study protocol for a randomised controlled trial. Trials. 2013 Jul;14(1):200.

16 Bhole R, Goyal N, Nearing K, Belayev A, Doss VT, Elijovich L, et al. Implications of limiting mechanical thrombectomy to patients with emergent large vessel occlusion meeting top tier evidence criteria. J Neurointerv Surg. 2017 Mar;9(3):225-8.

17 Goyal N, Tsivgoulis G, Frei D, Turk A, Baxter B, Froehler MT, et al. A multicenter study of the safety and effectiveness of mechanical thrombectomy for patients with acute ischemic stroke not meeting top-tier evidence criteria. J Neurointerv Surg. 2018 Jan;10(1):10-6.

18 Mokin M, Pendurthi A, Ljubimov V, Burgin WS, Siddiqui AH, Levy EI, et al. ASPECTS, Large Vessel Occlusion, and Time of Symptom Onset: Estimation of Eligibility for Endovascular Therapy. Neurosurgery. 2018 Jul; 83(1):122-7.

19 Vanacker P, Lambrou D, Eskandari A, Mosimann PJ, Maghraoui A, Michel P. Eligibility and Predictors for Acute Revascularization Procedures in a Stroke Center. Stroke. 2016 Jul;47(7):1844-9. 